
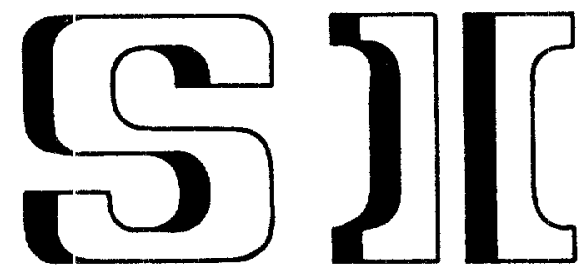

GSI-Preprint-98-33 Juli 1998

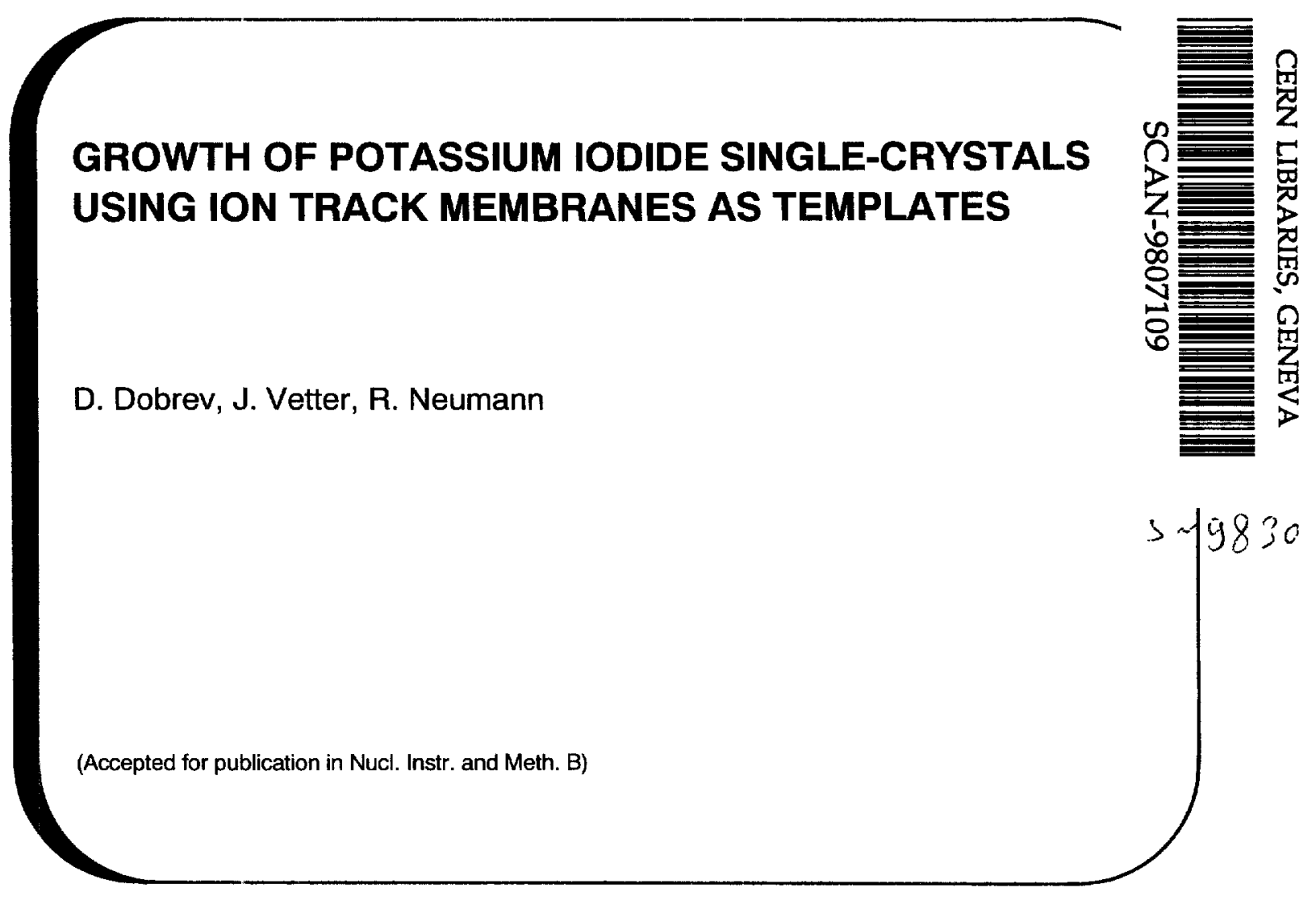

Gesellschaft für Schwerionenforschung $\mathrm{mbH}$ Planckstraße 1 - D-64291 Darmstadt - Germany Postfach 110552 - D-64220 Darmstadt • Germany 


\title{
Growth of Potassium Iodide Single-Crystals Using Ion Track Membranes as Templates
}

\author{
D.Dobrev", J.Vetter and R.Neumann \\ Gesellschaft für Schwerionenforschung (GSI), Planckstr.1, D-64291 Darmstadt, \\ Germany
}

\begin{abstract}
Summary
A principle possibility is demonstrated to apply the ion track membranes as a template for the crystallization of inorganic salts. As an example, potassium iodide has been grown in a matrix of etched ion tracks produced in polycarbonate foils. Arrays of stable free-standing cylindrical microcolumns are observed after dissolution of the organic matrix. They represent single crystals oriented with their $<100>$ or $<110>$ crystallographic directions along the cylinder axes. Possible ways to govern their predominant orientations are briefly discussed.
\end{abstract}

Keywords: ion, track, membrane, crystal growth, potassium iodide. PACS'96 Codes: 61.50-f, 61.82.-d, 81.10.-h, 81.15.Lm.

\section{Introduction}

The current interest in microscale structures is stimulated by the continuous process of miniaturisation mainly in microelectronics. Also, these objects may display novel material properties, largely as a result of their limited size[1-3]. Therefore, the development of new preparation methods of small particles including microcrystals with defined parameters is actual for the fabrication of miniature electronic components and new composite materials.

The use of etched ion tracks in organic foils and other insulating materials as templates is of particular interest for the fabrication of micro- and nanosized structures [3-7]. The ion track membranes have been already used in the past for the deposition of thin metal wires by electroplating [8-12]. Principally, the etched ion track channels might be also employed for the matrix crystallization of different inorganic salts.

In the present work, the growth of KI single crystals is demonstrated in etched ion tracks in polymer foils which served as templates during the crystallization process. KI is a salt that forms regular crystals. It is very well soluble in water and readily crystallizes at normal conditions. Therefore, its growth behaviour was chosen as a model in this investigation.

\footnotetext{
' GSI guest from the Inst. of Phys. Chemistry, Bulg. Acad. of Sci., Sofia 1040, Bulgaria.

Paper presented at SHIM'98, Berlin, Germany, 11-15 May 1998. Accepted for publication in NIM B.
} 
between two cubic planes intersecting under an angle of $90^{\circ}$, that corresponds to a $<110>$ orientation. Crystals oriented in such a way have been observed in small regions of some samples, an example being presented in Fig.4.a. This figure indicates that the KI crystals are mutually oriented along the other two of their crystallographic axes as well.

The oriented growth of KI crystals in the etched ion tracks can be stipulated by various reasons. If the initial crystallization starts from randomly oriented nuclei, the geometrical selection will take place due to the anisotropy of the crystal growth. The grain that has the most favourable orientation will overgrow the rest of the grains, and finally the most loosely packed direction will predominate. In the present case it can be the $\langle 110\rangle$ orientation, but never a two-axial one. The growth of the KI crystals in our experiments is obviously of epitaxial nature. Their orientations entirely depend on the orientation of the KI crystals that were at first formed on the surface of the polymer membrane. The assumption of an epitaxial mode of growth is supported by the two-axial orientation of the crystals in Fig.4b.

However, the formation of oriented crystals on a sufficiently smooth and crystallographically indifferent substrate depends on the conditions of deposition. According to the studies of electrocrystallization [13-15] and physical vapour deposition of metals on amorphous substrates [16-18], the orientation of the crystallites in deposited layers depends on the overvoltage during deposition in the first case and on the rate of deposition in the second case. Thus, in both cases the formation of textured claddings is determined by the supersaturation at which the initial growth is carried out. Therefore, it should be expected, that by varying the parameter of supersaturation, one will also affect the orientation of the crystals deposited in the nuclear pores. The application of suitable single-crystalline substrates that can provide conditions for an epitaxial growth is of great interest as well.

During crystallization of $\mathrm{KI}$ in the etched ion tracks a morphological instability has been frequently observed if the track diameter exceeded $5 \mu \mathrm{m}$. Fig. 8 shows such a morphological instability on the $<100>$ oriented crystals. It can lead to the formation of hollow cylindrical crystals as illustrated in Fig.9.

The KI crystals of micrometer size have displayed some plastic properties. They can be mechanically bent after the dissolution of the organic matrix as demonstrated in Fig. 10.

Finally, similar to $\mathrm{KI}$, we observed that the crystal growth of some other salts $(\mathrm{KCl}$, Seignette salt $-\mathrm{C}_{4} \mathrm{H}_{4} \mathrm{KNaO}_{6}, \mathrm{CuSO}_{4}$ ) showed the same features in the etched ion tracks.

In conclusion, ion track membranes can be applied as templates for the fabrication of oriented single crystals with micrometer dimensions from different inorganic salts. 


\section{Experimental}

Polycarbonate foils (Makrofol N, Bayer, Leverkusen), $60 \mu \mathrm{m}$ thick and $50 \mathrm{~mm}$ in diameter, were irradiated normal to the surface with ${ }^{238} \mathrm{U}$ ions accelerated up to 11.4 $\mathrm{MeV} / \mathrm{u}$ at the UNILAC linear accelerator of GSI, until the density of ion tracks reached $1 \times 10^{6}$ ions $/ \mathrm{cm}^{2}$. Subsequently, the foils were etched in $6 \mathrm{~mol} / 1 \mathrm{NaOH}$ containing $10 \% \mathrm{CH}_{3} \mathrm{OH}$ at $50^{\circ} \mathrm{C}$. By choosing two different etching times of appropriate length, we produced channels with diameters of approximately 2.5 and 5 $\mu \mathrm{m}$, respectively.

The crystallization of KI was carried out from saturated KI water solutions in Petri dishes with a diameter of $60 \mathrm{~mm}$. The experimental set-up is shown in Fig. 1. The etched foils were left to float on the surface of the saturated solution as seen in Fig. Ia, and the dishes were kept uncovered at room temperature. The KI solution crept out trough the ion track pores on the top surface of the foil and its concentration increased by the evaporation of the solvent. The crystallization of KI started when a certain supersaturation had built up. Further growth of the solid phase was maintained by water evaporation from both the foil surface and from the free exterior region of the KI solution. Within 15 hours the foils have been covered on their top sides by numerous comparatively large crystals of KI. The etched channels have been also filled with KI as shown in Fig.1b. The foils were removed from the solution and airdried on filter paper. Afterwards the polycarbonate matrixes were dissolved in $\mathrm{CH}_{2} \mathrm{Cl}_{2}$. The residual crystallites were used for the preparation of samples for microscopic investigation. The bottom sides of these crystallites facing the removed organic material were metallized by sputtering with a thin conductive gold layer. The samples were studied by means of a scanning electron microscope (Philips XL 30).

\section{Results and Discussion}

KI crystals have a face-centred cubic (FCC) lattice. The reticular density of the various crystallographic planes of this lattice decreases in the sequence (111), $(100),(110)$, etc. The most close-packed planes are those that should be observed in the crystal forms if the growth conditions are close to equilibrium. KI crystals that have been grown free onto the foil surface are shown in Fig. 2. They are well-formed with a cubic habitus.

The KI crystals grown in the etched ion tracks with a diameter of about $2.5 \mu \mathrm{m}$ are demonstrated at low magnification in Fig. 3.

In the tracks, the growth propagates only along the axes of the pores, since it is limited in the perpendicular directions by the walls of the cylindrical templates. Therefore, these crystals usually take on a cylindrical shape as can be seen in Fig 4 . Nevertheless, in some cases faceting is also observed parallel to the long axes of the crystals (see Fig.5). That means that the lateral growth of the crystals can stop when their edges contact the walls of the pores.

However, the growing front is always formed by true crystallographic facets. The arrangement of these facets allows one to determine the crystallographic axes, i.e. the orientation of the growing crystals. In Fig. 6 the top crystallographic plane of the crystal with a diameter of $5.6 \mu \mathrm{m}$ is obviously a (100) plane, that confirms the orientation of this crystal along the $\langle 100\rangle$ axis. The same orientation is characteristic of the crystals imaged in Fig.4a. It dominates in most of experiments that have been carried out at the conditions described above. In Fig.7 a crystal of approximately the same diameter is shown. The growing end of this crystal exhibits in its centre an edge 


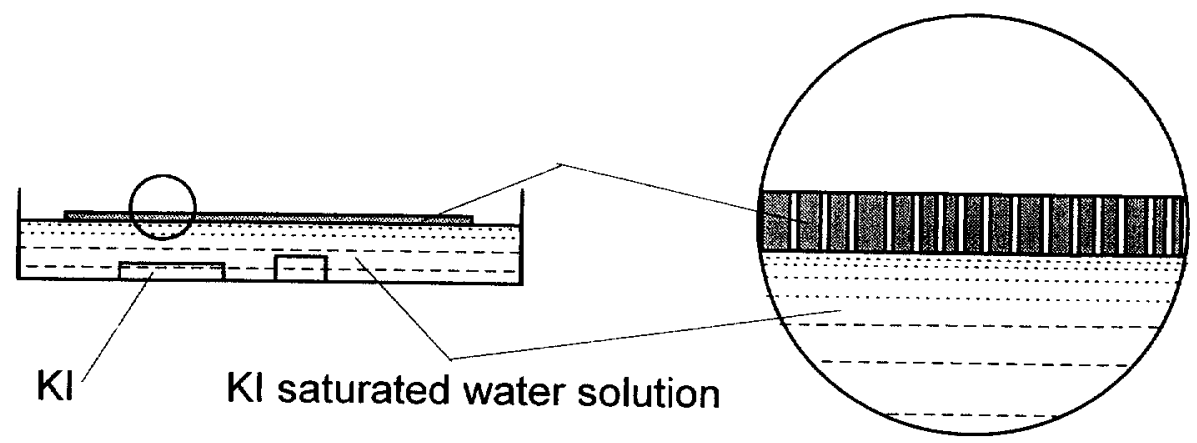

(a)

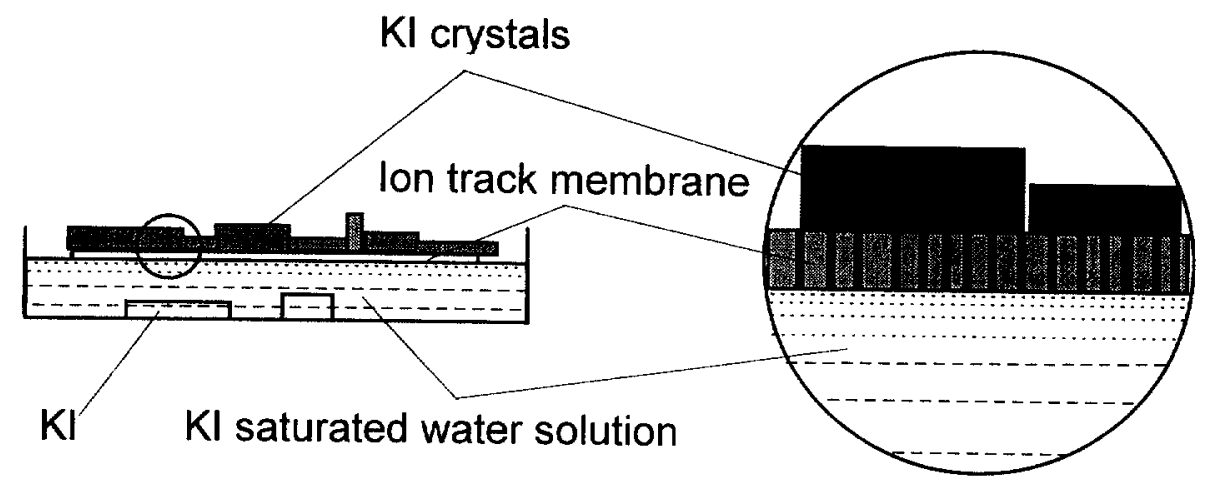

(b)

Fig.1. 


\section{References}

[1] G.A. Ozin, Adv. Mater. 4 (1992) 612.

[2] M. Sundaram, S.A. Chalmers, P.F. Hopkins and A.C. Gossard, Science 254 (1991) 1326.

[3] K.D. Wise and K. Najafi, Science 254 (1991) 1335.

[4] R. Spohr, Ion Tracks and Microtechnology, Vieweg, Braunschweig, 1990.

[5] T.M. Whitney, J.S. Jiang, P.C. Searson and C.L. Chien, Science 261 (1993) 1316.

[6] D.A. Mawlawi, M. Moskovits, D.S. Ellis, A. Williams and J.M. Xu, Proc. Int. Semicond. Dev. Res. Symp., College Station, TX, July 1-4, 1993, p.311.

[7] C. Martin, Science 266 (1994) 1961.

[8] G.E. Possin, Rev. Sci. Instr. 41 (1970) 772.

[9] W. Williams and N. Giordano., Rev. Sci. Instr. 55 (1984) 410.

[10] R. Penner, C. Martin, Anal. Chem. 59 (1987) 2625.

[11] S. Chakarvarti and J. Vetter, Nucl. Instr. Meth. B62 (1991) 109.

[12] D. Dobrev, J. Vetter and N. Angert, GSI Rep.-1995, p.261, 1996

[13] N. Pangarov, and D. Dobrev, Comp. Rend. Acad. Bulg. Sci, 15 (1962) 519.

[14] N. Pangarov, and D. Dobrev, Comm. of the Inst of Phys. Chem, 2 (1963) 101.

[15] N.Pangarov, D.Sc. Thesis, Sofia,. 1967

[16] D. Dobrev, Proc.Vth Int. Conf. Text. Mat., March 28-31, 1978, Aachen, p. 171.

[17] D.Dobrev and A.Gittis, J.Cryst.Growth, 52 (1981) 159.

[18] E.Grantscharova and D. Dobrev, Thin Solid Films, 161 (1988) 213.

\section{FIGURE CAPTIONS}

Fig.1. Experimental set-up: a) Starting position, b) End of the experiment.

Fig.2. KI crystals grown free on the polycarbonate foil.

Fig.3. An array of KI crystals grown in etched ion track pores with diameter of $2.4 \mu \mathrm{m}$.

Fig.4. Cylindrical KI crystals of different crystal orientation grown in pores with diameter of $2.4 \mu \mathrm{m}$.
a) $<100>$ orientation, b) $<110>$ orientation.

Fig.5. Polygonized KI crystals wit diameter of $2.4 \mu \mathrm{m}$.

Fig.6. KI single crystal with a diameter of $5.6 \mu \mathrm{m}$ with a (100) plane parallel to the growing front.

Fig.7. KI single crystal with a diameter of $5.6 \mu \mathrm{m}$ with two cubic planes intersecting under $90^{\circ}$ angle in its centre tantamount to $<110>$ orientation.

Fig.8. Morphological instability of the growth of $<100>$ oriented crystals with diameter of $5.6 \mu \mathrm{m}$.

Fig.9. A hollow KI crystal.

Fig.10. KI crystals mechanically bent after the dissolution of the organic matrix. 


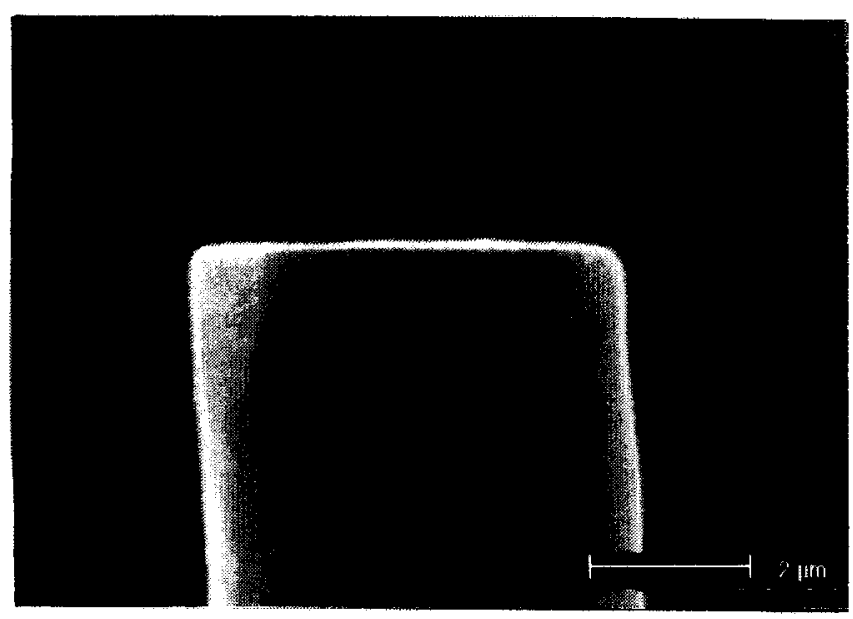

Fig.6.

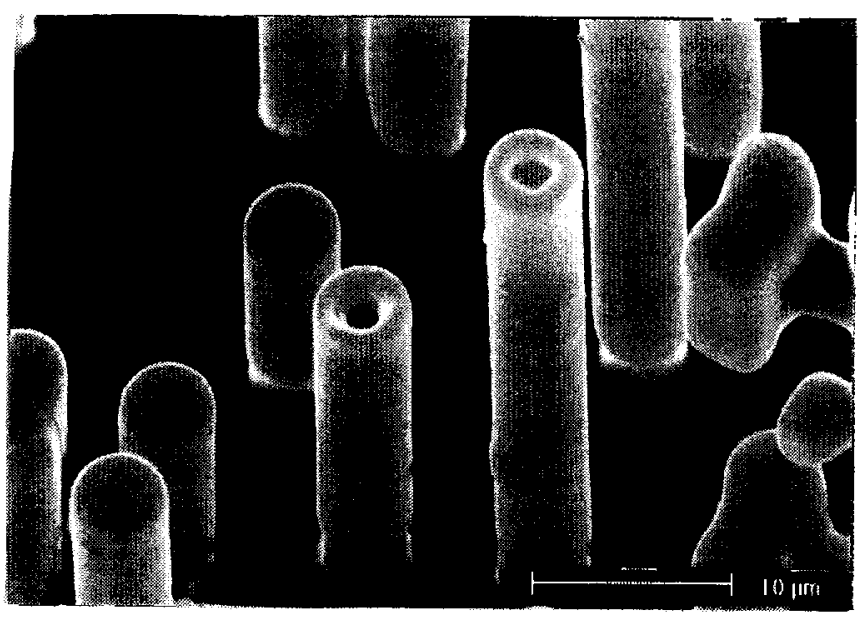

Fig.8.

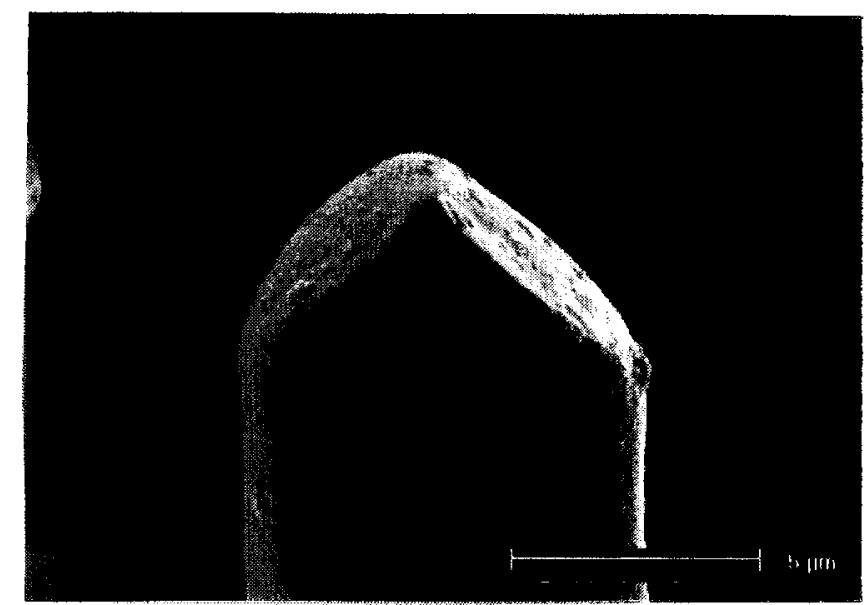

Fig.7.

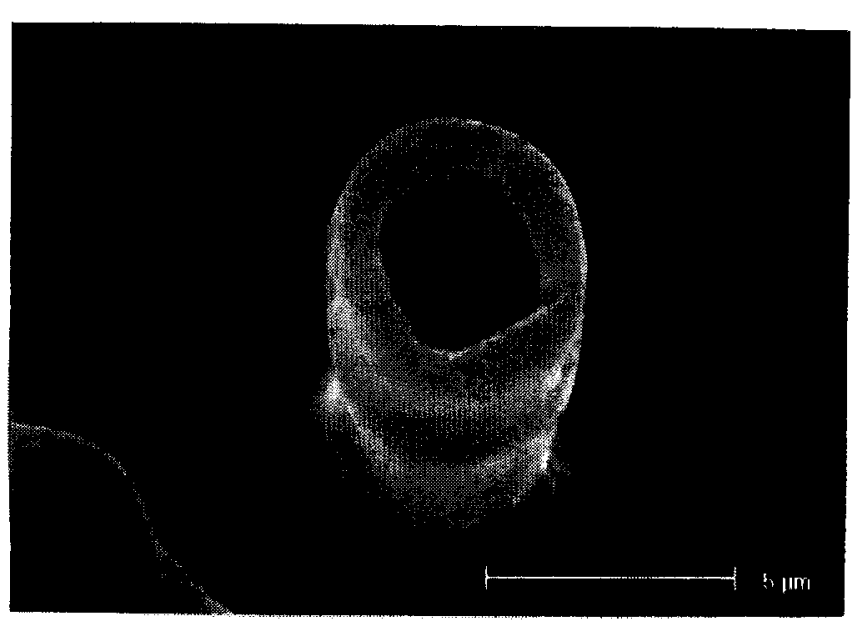

Fig.9.

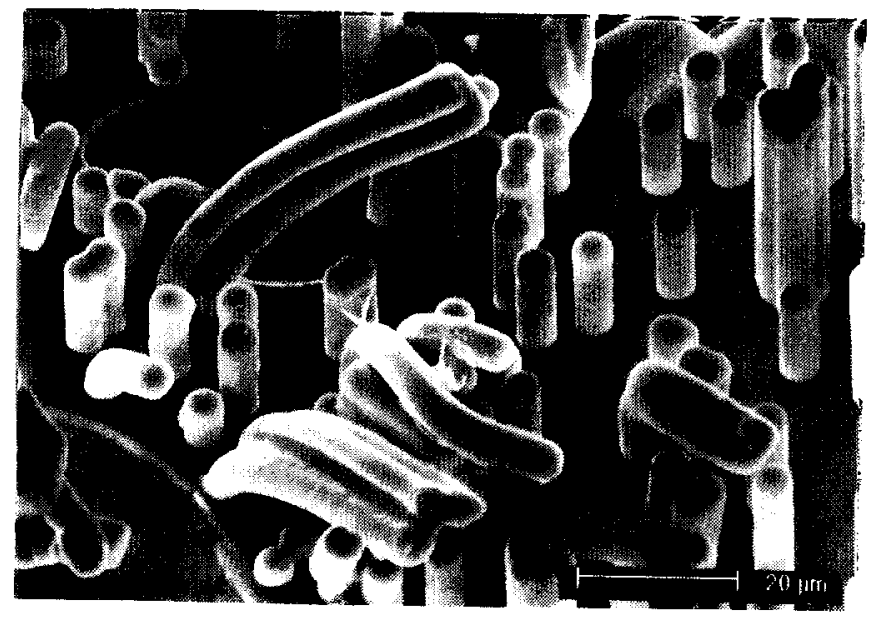

Fig.10. 
